\title{
An Algorithm for Consensus Trees
}

\author{
Pongsaphol Pongsawakul \\ pongsaphol@pongsaphol.com
}

March 9, 2020

\begin{abstract}
We consider the tree consensus problem, an important problem in bioinformatics. Given a rooted tree $t$ and another tree $T$, one would like to incorporate compatible information from $T$ to $t$. This problem is a subproblem in the tree refinement problem called the RF-Optimal Tree Refinement Problem defined by in Christensen, Molloy, Vachaspati and Warnow [WABI'19] who employ the greedy algorithm by Gawrychowski, Landau, Sung, and Weimann [ICALP'18] that runs in time $O\left(n^{1.5} \log n\right)$. We give a faster algorithm for this problem that runs in time $O(n \log n)$. Our key ingredient is a bipartition compatibility criteria based on amortized-time leaf counters. While this is an improvement, the fastest solution is an algorithm by Jansson, Shen, and Sung [JACM'16] which runs in time $O(n)$.
\end{abstract}

\section{Introduction}

We consider the tree consensus problem, an important problem in bioinformatics. Given a rooted tree $t$ and another rooted tree $T$, we would like to combine "information" from $T$ into $t$. More over, we would like to only greedily take information that is currently consistent with our current $t$. (See definitions below.) This problem is a subproblem in the tree refinement problem called RF-Optimal Tree Refinement Problem defined by in Christensen, Molloy, Vachaspati and Warnow [1] who employ the greedy algorithm by Gawrychowski, Landau, Sung, and Weimann [2] that runs in time $O\left(n^{1.5} \log n\right)$. We give a faster algorithm for this problem that runs in time $O(n \log n)$. Our key ingredient is a bipartition compatibility criteria based on amortizedtime leaf counters. While this is an improvement, the fastest solution is an algorithm by Jansson, Shen, and Sung [3] which runs in time $O(n)$.

The algorithm by Gawrychowski et al 2] works in a more general case where the goal is the find the greedy consensus trees from $k$ trees. In this case, their algorithm runs in time $O\left(k n^{1.5} \log n\right)$, an improvement over $O\left(k n^{2}\right)$ of Jansson et al [3]. For this problem, Sung [4] also present an algorithm that runs in time $O\left(k^{2} n\right)$, improving over Gawrychowski et al 2] when $k=O(\sqrt{n} \log n)$.

In an earlier version, we erroneously claimed that our algorithm works for the case with many trees. We thank Pawel Gawrychowski and Oren Weiman for pointing this out. Jittat Fakcharoenphol who help advising the author on this manuscript would like to take the full responsibility for this mistake.

\section{Definitions}

We start with definitions related to trees and consistency.

Let $V(T), E(T)$ and $R(T)$ be vertex set, edge set and the root of tree $T$. For every vertex $u \in V(T)-$ $\{R(T)\}$, let $\operatorname{par}(u)$ be parent node of node $u$. For every vertex $u \in V(T)$, let $\operatorname{depth}(u)$ be depth of node $u$. We can denote as $\operatorname{depth}(u)=\operatorname{depth}(\operatorname{par}(u))+1$, for every vertex $u \in V(T)-\{R(T)\}$ and $\operatorname{depth}(R(T))=1$. For every vertex $u \in V(T)$, let $L(u)$ be set of all leaves on subtree $u$. Let size $(u)=|L(u)|$ for each $u \in V(T)$. For each node $u \in V(T)-\{R(T)\}$, we call $\Lambda(u)$ be set of bipartition at edge $(u, p a r(u))$. For each bipartition, $\Lambda(u)$, we can represent into two clusters, $A=L(u)$ and $B=L(R(T))-L(u)$, and denoted by $A \mid B$. The set of 
bipartitions of $T$ can denoted by $C(T)=\{\Lambda(u): u \in V(T)-\{R(T)\}\}$. Let $R F\left(T_{a}, T_{b}\right)$ be Robinson-Foulds distance between trees $T_{a}$ and $T_{b}$. RF-distance can denoted by $R F\left(T_{a}, T_{b}\right)=\left|C\left(T_{a}\right)-C\left(T_{b}\right)\right|$.

The set $S$ of bipartitions is compatible if there exists tree $T$ such that $C(T)=S$. leaves set $A$ is compatible with $t^{\prime}$ when have node $u$ that for all $v \in \operatorname{child}(u)$ if only if $L(v) \cap S=\emptyset$ or $L(V) \subseteq S$.

\section{The $O\left(n^{2}\right)$ algorithm}

In this section, we describe a simpler version of the algorithm and prove its correctness and its running time (in Subsection 3.1). We improve its running time in Section 4

We assume that both $T$ and the current $t^{\prime}$ are equipped with a data structure that given an id of a bipartition $b$, find vertex $u$ in the trees such that $\Lambda(u)=b$. Since $t^{\prime}$ changes over time, we assume that our data structures can handle the tree update efficiently. We discuss this in Subsection 3.2

The main loop of the algorithm iterates over all bipartition of $T$ recursively and, if possible, add each bipartition to $t^{\prime}$.

We define variables used in the main loop. Let $z$ be the node that have minimum depth in tree $t^{\prime}$ for each bipartition in $T$. The main loop is described below.

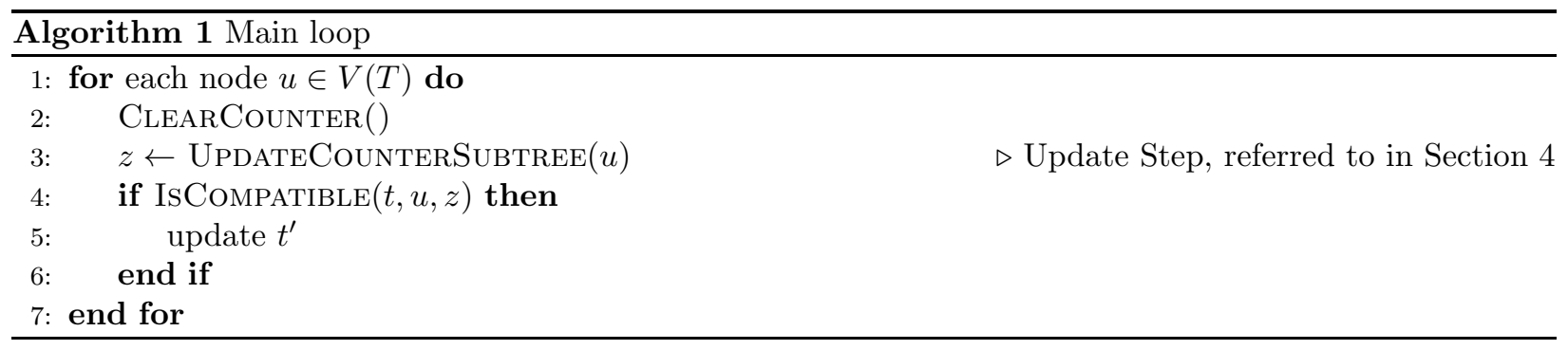

The main loop uses the following function.

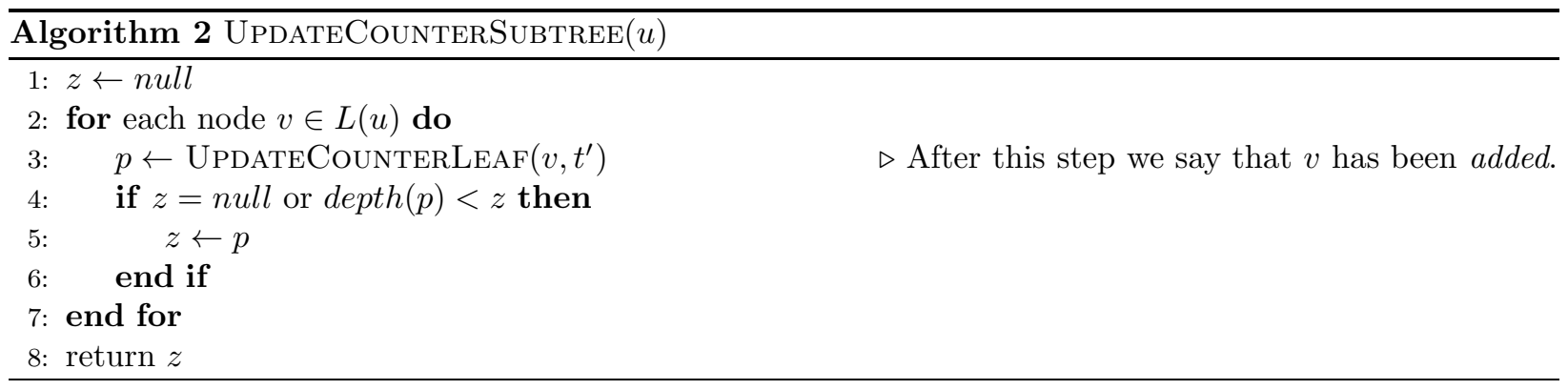

Our algorithm maintains variable counter for each vertex in $t^{\prime}$. We also keep a list of dirty vertices so that ClearCounter can run in $O(1)$ time. Variable counter is updated in function UpdateCounterleaf. Note that counter changes over time as UpdateCounterSubtree keeps adding leaves in $L(u)$. The algorithm ensures that counter $(u)$ is exactly as follows. If $u$ is a leaf vertex, we let

$$
\operatorname{counter}(u)= \begin{cases}1 & \text { if } v \text { has been added } \\ 0 & \text { otherwise }\end{cases}
$$

For other internal vertex $u$, we let

$$
\operatorname{counter}(u)=\sum_{v \in \operatorname{child}(u)} \begin{cases}\text { counter }(v) & \text { if counter }(v)=|L(v)| \\ 0 & \text { otherwise }\end{cases}
$$


For each call this function, it can take amortized $O(1)$ time (to be proved later). The following algorithm describes function UpdateCounterLeaf.

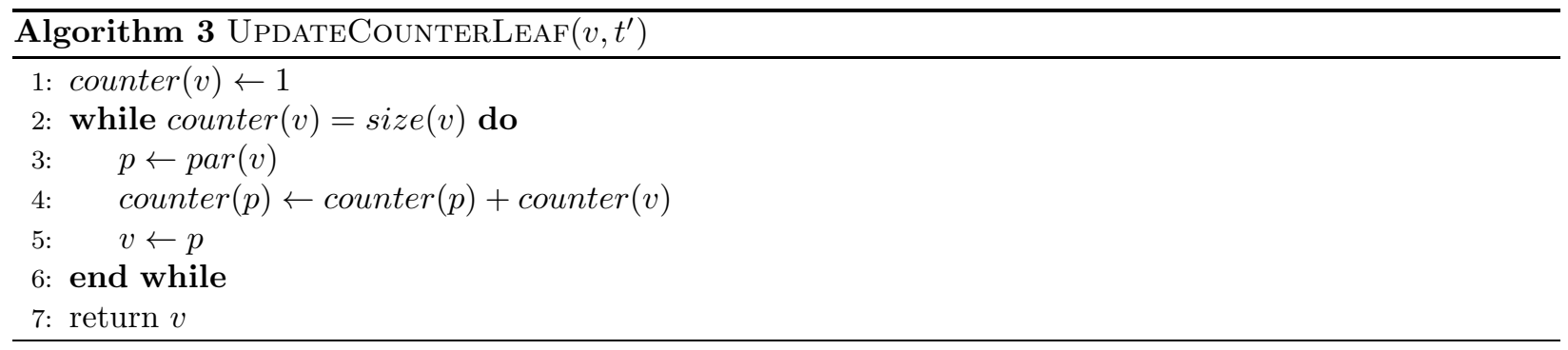

Next, we have algorithm that check that add from previous algorithm is compatible to $t^{\prime}$. We can track the node $u$ that have minimum depth and have counter $(u)>0$ in the while loop at the previous algorithm.

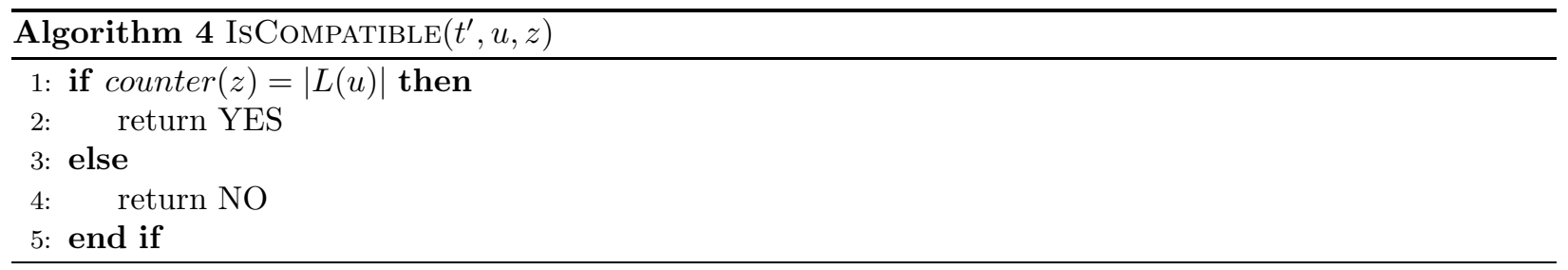

We prove the correctness of the algorithm. We note that it considers all bipartitions.

Lemma 1. The main loop considers all bipartitions in $T$.

Proof. From definition, $\Lambda(u)$ can represent all bipartitions of $T$ and for each bipartition, we have consider all node in $\Lambda(u)$. From this, we can consider all bipartitions in $T$.

For clarity, for each vertex $w \in t^{\prime}$ we denote by $L_{t^{\prime}}(w)$ its leaf set in $t^{\prime}$. Note each call to UpdateCounTERLEAF increases counter for each vertex by at most 1 , and we call UPDATECOUNTERLEAF exactly $|L(u)|$ times. This implies the next lemma, which can be formally proven by induction.

Claim 1. During the call of $\operatorname{UpdateCounterSubtree}(u)$, for any vertex $v \in t^{\prime}$, counter $(v) \leq \mid L(u) \cap$ $L_{t^{\prime}}(v) \mid$. Moreover, if $L_{t^{\prime}}(v) \subseteq L(u)$, counter $(v)=\left|L(u) \cap L_{t^{\prime}}(v)\right|=\left|L_{t^{\prime}}(v)\right|$, i.e., the counter attains its maximum.

The following the key lemma.

Lemma 2. Let $z^{\prime}$ be the least common ancestor of leaf vertices in $L(u)$ in $t^{\prime}$. After UPDATECounterSubTREe $(u)$ is called, leaf set $L(u)$ is compatible with the current $t^{\prime}$ if and only if counter $\left(z^{\prime}\right)=|L(u)|$.

Proof. Note that after the call to UpdateCounterSubtree $(u)$, we have called UpdateCounterLeaf $(v)$ for every leaf $v$ in $L(u)$. From Claim 1, we only need to consider the case when $\operatorname{counter}\left(z^{\prime}\right) \leq|L(u)|$.

The algorithm maintains vertex $z$, which is the vertex closest to the root that UPDATECOUNTERLEAF has touched. We first consider the case that $z=z^{\prime}$.

We show that if counter $\left(z^{\prime}\right)=|L(u)|$, then for each child $w \in \operatorname{children}\left(z^{\prime}\right), L_{t^{\prime}}(w) \cap L(u)=\emptyset$ or $L_{t^{\prime}}(w) \subseteq L(u)$. This implies that $L(u)$ is compatible with $t^{\prime}$.

We note that $z^{\prime}$ is not a leaf. Consider each $w \in \operatorname{children}\left(z^{\prime}\right)$. We only need to consider $w$ such that $L_{t}(w) \cap L(u) \neq \emptyset$. The only way counter $\left(z^{\prime}\right)=|L(u)|$ is when $w$ is "complete", i.e., counter $(w)=\left|L_{t^{\prime}}(w)\right|$. Since counter $(w) \leq\left|L(u) \cap L_{t^{\prime}}(w)\right|$ (from Claim 1), we know that $L_{t^{\prime}}(w) \subseteq L(u)$.

On the other hand, if $L(u)$ is compatible with $t^{\prime}$, we show that counter $\left(z^{\prime}\right)=|L(u)|$. We prove a stronger statement: if $L(u)$ is compatible with $t^{\prime}$ for every vertex $v \in t^{\prime}$ in the subtree rooted at $z^{\prime}$,

$$
\text { counter }(v)=\left|L(u) \cap L_{t^{\prime}}(v)\right|,
$$


i.e., the upper bound in Claim 1 attains its maximum. To do so, we prove inductively on the structure of $t^{\prime}$. Clearly, the claim is true when $v$ is a leaf. Consider vertex $v \neq z^{\prime}$ in the subtree of $t^{\prime}$ rooted at $z^{\prime}$. If $L_{t^{\prime}}(v) \cap L(u)=\emptyset$, counter $(v)=0$; thus the property follows. Now, consider $v$ such that $L_{t^{\prime}}(v) \cap L(u) \neq \emptyset$. Let $w$ be a child of $z^{\prime}$ such that $v$ belongs to subtree rooted at $w$. Since $L_{t^{\prime}}(v) \cap L(u) \neq \emptyset$ and $L_{t^{\prime}}(v) \subseteq L_{t^{\prime}}(w)$, we know that $L_{t^{\prime}}(w) \cap L(u) \neq \emptyset$. Since $L(u)$ is compatible with $t^{\prime}$, we have that

$$
L_{t^{\prime}}(w) \subseteq L(u),
$$

implying that $L_{t^{\prime}}(v) \subseteq L(u)$; thus counter $(v)=\left|L_{t^{\prime}}(v)\right|=\left|L_{t^{\prime}}(v) \cap L(u)\right|$, from Claim 1 ,

Finally, consider $z^{\prime}$. Note that since $z^{\prime}$ is the common ancestor of leaves in $L(u), L_{t^{\prime}}\left(z^{\prime}\right) \supseteq L(u)$. For each child $w$ of $z^{\prime}$, when $L_{t^{\prime}}(w) \cap L(u) \neq \emptyset$, w is complete and propagate $\left|L_{t^{\prime}}(w) \cap L(u)\right|$ to counter $\left(z^{\prime}\right)$. Summing all children of $z^{\prime}$, we have that counter $\left(z^{\prime}\right)=|L(u)|$.

This completes the proof of the lemma.

Lemma 3. Tree compatibility condition works

From above condition, our algorithm have add function counter $(u)$. for each subtree $L(u)$ have fully resolved when counter $(u)=|L(u)|$

\subsection{Running time analysis}

We first analyze the running time of the algorithm except the calls to UPdATECounterSubTREe. We show that this part runs in linear time.

We start with UpdateCounterLeaf.

Lemma 4. Function UpdateCounterLeaf $\left(v, t^{\prime}\right)$ runs in amortized $O(1)$ time.

Proof. We use the potential method. Our data structure consists of variables counter for all vertices in $t^{\prime}$. Denote the data structure at time $i$ by $D_{i}$. We say that a vertex $u \in t^{\prime}$ is incomplete if $0<\operatorname{counter}(u)<$ $|L(u)|$. Let potential function $\Phi\left(D_{i}\right)$ be the number of incomplete vertices in $t^{\prime}$ time $i$. Using the potential method, when the data structure changes from $D_{i-1}$ to $D_{i}$, the amortized cost of an operation is $\hat{c}=c+\Delta \Phi$, where $c$ is an actual cost, and $\Delta \Phi=\Phi\left(D_{i}\right)-\Phi\left(D_{i-1}\right)$. Let $D_{0}$ be initial data structure after CLEARCounter is called; thus $\Phi\left(D_{0}\right)=0$. Note that $\Phi\left(D_{i}\right) \geq \Phi\left(D_{0}\right)=0$ for any $i$.

When invoking UpdateCounterLeaf at time $i$, let $k$ be number of times the while loop in Lines 2 - 6 is executed. Clearly, the actual cost $c$ of the operation is $k+1$. Let $\Delta \Phi=\Phi\left(D_{i}\right)-\Phi\left(D_{i-1}\right)$.

We claim that $\Phi\left(D_{i}\right)=\Phi\left(D_{i-1}\right)-k+1$, i.e., the number of incomplete vertices decreases by $k-1$. Let $v^{\prime}$ be the actual leaf that UpdateCounterLeaf is called on. Note that each time the loop is executed, counter $(v)=\operatorname{size}(v)=|L(v)|$. Except when $v=v^{\prime}$, previously at time $i-1$, we know that $0<\operatorname{counter}(v)<$ $|L(v)|$, because $v$ is an internal vertex with at least 2 children; hence $v$ was incomplete at time $i-1$. Since counter $(v)=|L(v)|$ at time $i, v$ is no longer incomplete; thus the number of incomplete vertices decreases by $k-1$ as claimed.

Thus the amortized cost $\hat{c}=k+1+\Delta \Phi=k+1+(-k+1)=2=O(1)$.

We now analyze the running time of UpdateCounterSubtree.

Lemma 5. Function UpdateCounterSubtree $(u)$ runs in time $O(|L(u)|)$.

Proof. From Lemma 4, it is clear that UpdateCounterSubtree runs in time $O(|L(u)|)=O(n)$ and it is invoked for $O(n)$ time. Therefore, the total running time of the function is $O\left(n^{2}\right)$. Combining the two parts, we get that the algorithm runs in $O\left(n^{2}\right)$. 


\subsection{Updating $t$}

In this section, we show that when the bipartition defined by $L(u)$ is compatible with $t^{\prime}$, we can update $t^{\prime}$ to include that bipartition efficiently in time $\operatorname{deg}(u)$. When $t^{\prime}$ is compatible with a bipartition defined by $L(u)$ for $u \in T$, to update $t^{\prime}$ we have to create a new child of $z$ that consists of only children of $z$ that corresponds to the bipartition $L(u)$. Note that these children are those "full" counter that also propagate the counter to $z$. Therefore, when a child propagate a counter to any vertex, we keep a list of them. When we need to update $t^{\prime}$ at $z$, we can take every vertex in this list, and create a new child $z^{\prime}$ of $z$ with these vertices as $z^{\prime}$ 's children and update their counter accordingly. This can be done in time $O(\operatorname{deg}(u))$.

\section{The faster algorithm: heavy child optimization}

In this section, we describe a simple method to speed up the algorithm from the previous section. Note that the only bottle-neck to a nearly linear time algorithm is the counting procedure.

As a preprocessing, we assume that for each vertex $u \in T$, we know $|L(u)|$. This can be computed in $O(n)$ time. The improved algorithm is described below.

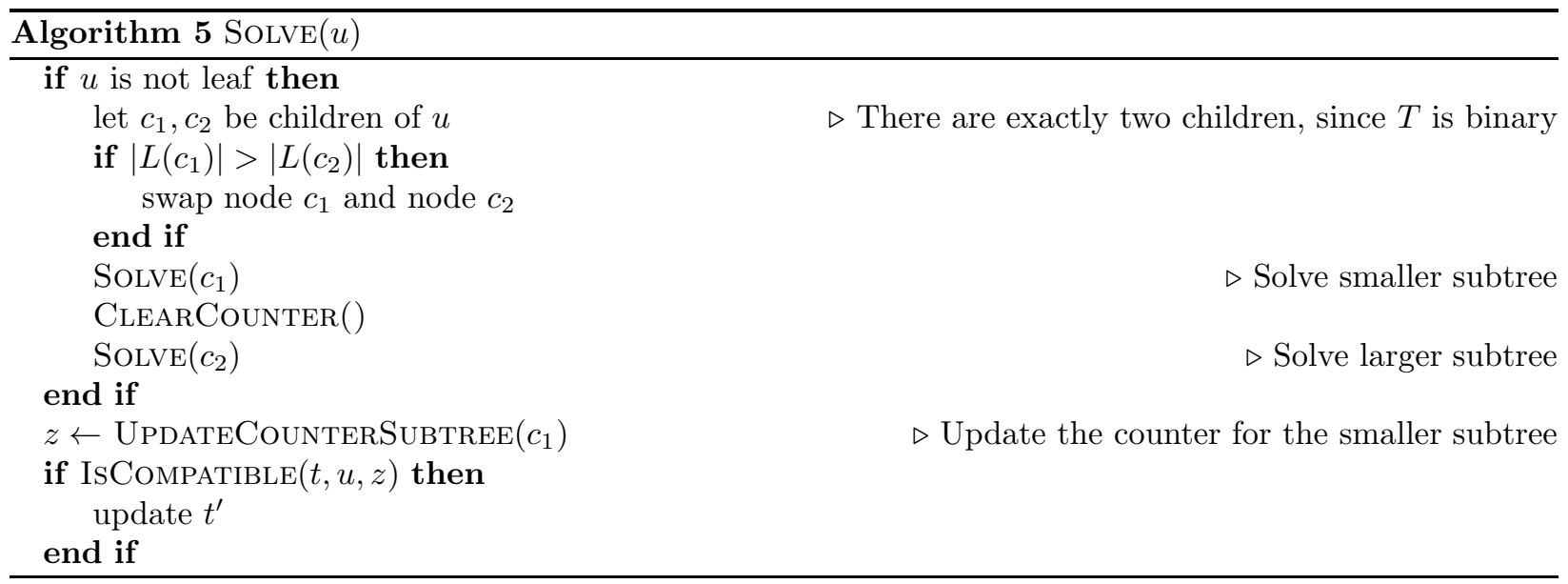

To see that this algorithm is the correct implementation of the Main loop, we essentially need to show that at the end of $\operatorname{Solve}(u)$, variable counter is exactly equal to variable counter right after the "Update Step" in Line 3 in the Main loop while processing $u$, i.e., variable counter is exactly equal to the case when every leaf in $L(u)$ has been added while no other leaves have been added. This can be shown by induction on the calls of SOLVE. We omit the proof in this version of the manuscript.

We are left to analyze its running time.

Theorem 1. The algorithm Solve runs in $O(n \log n)$ time.

Proof. Note that the running time for all other operations in Solve is $O(1)$ per invocation. Since Solve is called for $O(n)$ time, the total running time of these operations is $O(n)$. Also, the running time of ClearCounter () can be amortized to the running time of UpdateCounterSubtreE, where the counters are updated.

Therefore, we are left to analyze the running time of UPDATECOUNTERSUBTREE.

Note that $\operatorname{UpdateCounterSubtree}(u)$ for $u \in T$ runs in time linearly in the number of leaves, $|L(u)|$, from Lemma 5. Hence, we can charge the cost to these leaves.

We analyze the running time by counting the number of times each leaf is involved in this charging scheme. Note that we only call UPDATECOUNTERSuBtree at $c_{1}$, which is the lighter subtree. Clearly, each leaf $u$ belongs to at most $O(\log n)$ light subtrees; hence, it is charged by at $\operatorname{most} O(\log n)$ time. Summing all leaves, we have that the total running time for UPDATECOUNTERSuBtree is $O(n \log n)$. 


\section{Acknowledgements}

We would like to thank Pawel Gawrychowski and Oren Weiman for pointing out our erroneous claim and also give us reference to Sung's result [4]. As mentioned earlier, Jittat Fakcharoenphol who help advising the author on this manuscript would like to take full responsibility for this mistake. We would like to thank Jittat Fakcharoenphol for suggesting this problem to work on and for his help in editing this manuscript.

\section{References}

[1] Sarah Christensen, Erin K. Molloy, Pranjal Vachaspati, and Tandy Warnow. TRACTION: Fast NonParametric Improvement of Estimated Gene Trees. In Katharina T. Huber and Dan Gusfield, editors, 19th International Workshop on Algorithms in Bioinformatics (WABI 2019), volume 143 of Leibniz International Proceedings in Informatics (LIPIcs), pages 4:1-4:16, Dagstuhl, Germany, 2019. Schloss Dagstuhl-Leibniz-Zentrum fuer Informatik.

[2] Pawel Gawrychowski, Gad M. Landau, Wing-Kin Sung, and Oren Weimann. A faster construction of greedy consensus trees. In Ioannis Chatzigiannakis, Christos Kaklamanis, Dániel Marx, and Donald Sannella, editors, 45th International Colloquium on Automata, Languages, and Programming, ICALP 2018, July 9-13, 2018, Prague, Czech Republic, volume 107 of LIPIcs, pages 63:1-63:14. Schloss Dagstuhl - Leibniz-Zentrum für Informatik, 2018.

[3] Jesper Jansson, Chuanqi Shen, and Wing-Kin Sung. Improved algorithms for constructing consensus trees. J. ACM, 63(3), June 2016.

[4] Wing-Kin Sung. Greedy consensus tree and maximum greedy consensus tree problems. In Gautam K. Das, Partha S. Mandal, Krishnendu Mukhopadhyaya, and Shin-ichi Nakano, editors, WALCOM: Algorithms and Computation, pages 305-316, Cham, 2019. Springer International Publishing. 